\title{
Integration of polymeric membrane/dielectric sphere assemblies in microfluidic chips for enhanced-contrast imaging with low-magnification systems
}

\author{
Vittorio Viri $\odot{ }^{\dagger}$ Daniel Migliozzi $\odot,{ }^{\dagger}$ and Martin A. M. Gijs $\odot^{*}$ \\ Ecole Polytechnique Fédérale de Lausanne, Laboratory of Microsystems, Lausanne, Switzerland
}

\begin{abstract}
Current microscopy systems for the imaging of microorganisms are expensive because of their optimized design toward resolution maximization and aberration correction. In situations where such an optimization is not needed, for instance to merely detect the presence of pathogens in liquids for on-site analyses, a potential approach is to use highly refractive spheres in combination with low-magnification objectives to increase the resolution and the sensitivity of the optical sensing system in a cost-effective fashion. Indeed, for point-of-need assays, integration of optical elements on a microfluidic device can bring several advantages, such as test parallelization/automation and low-volume consumption. We report a study on $\mathrm{BaTiO}_{3}$ spheres that are partially embedded in thin polymeric membranes of mismatched refractive index. We computed the transformation that the polymeric membrane/dielectric sphere assembly (PMDSA) mediates on the light originating from the sample toward the optical detector and shows its enhanced-detection potential for a low-magnification objective. We then propose a method to easily fabricate chips with custom designs and precise location of such dielectric spheres relative to the microfluidic structures for enhanced imaging of microorganisms. We applied this concept to the detection of living fluorescent bacteria, either flowing in aqueous medium or immobilized in hydrodynamic traps. We quantified the contrast gain provided by the PMDSA for short exposure when used with a low-magnification objective. By comparing with a high-magnification objective, we also show how longer-term imaging can be still reliably performed with a more cost-effective system. Since the present PMDSA concept combines the optical enhancement of low-magnification systems with the flexibility of microfluidic handling, it can be highly suitable for portable and cost-effective systems for on-site analysis, from flow cytometry to longer-term antibiotic testing. (c) The Authors. Published by SPIE under a Creative Commons Attribution 4.0 Unported License. Distribution or reproduction of this work in whole or in part requires full attribution of the original publication, including its DOI. [DOI: 10.1117/1.JOM.1.1.014001]
\end{abstract}

Keywords: dielectric sphere; thin membrane; live-cell imaging; optics-integrated microfluidics.

Paper 20007 received Aug. 20, 2020; accepted for publication Oct. 29, 2020; published online Jan. 26, 2021.

\section{Introduction: Dielectric Spheres in Optical Imaging and Integration of Optical Elements on Microfluidic Chips}

In the past decade, dielectric microspheres ( $\mu \mathrm{Ss}$ ) have been widely studied because of their properties of improving the performance of optical systems in a cost-effective fashion: the effective field of view and magnification created by the $\mu \mathrm{Ss}$ were characterized, ${ }^{1,2}$ and resolution analyses were also performed in the case of $\mu \mathrm{Ss}$ immersed in liquid media ${ }^{3}$ or elastomers. ${ }^{4}$ Such $\mu$ Ss were used for various applications, such as microtopography measurements, ${ }^{5,6}$ biological structure imaging, ${ }^{7-10}$ single-molecule detection, ${ }^{11}$ single-nanoparticle detection, ${ }^{12}$ and fluorescence correlation spectroscopy. ${ }^{13,14}$ Previous examples of technical implementation employed scanning probes, ${ }^{6,15}$ arrays,${ }^{16}$ or thin polymeric films ${ }^{17}$ to perform the imaging. Arraying was also used to enable detection of nano-objects in a flow. ${ }^{12}$ However, handling such small objects for imaging purposes can be prohibitive when willing to increase throughput and decrease fabrication costs and complexity. For instance, placing $\mu \mathrm{Ss}$ in precise locations with high yield can prove

\footnotetext{
*Address all correspondence to Martin A. M. Gijs, martin.gijs@epfl.ch

${ }^{\dagger}$ These authors equally contributed to this work.
} 
extremely hard. Moreover, if the $\mu$ Ss are not well positioned relative to the sample, imaging and detection may be hindered. ${ }^{3,16}$ For applications that target the detection of large objects (e.g., bacteria or cells) instead of small objects (e.g., biomolecule, nanoparticles or subcellular structures), a large depth-of-field is usually required. ${ }^{18,19}$ Additionally, for applications that require long-term imaging of living microorganisms, sample handling and immobilization often constitute a significant challenge. Several microfluidic approaches have been proposed to facilitate such analyses, e.g., single-cell arraying ${ }^{20}$ and single bacteria immobilization. ${ }^{21}$ Integrating microoptical elements with microfluidic platforms can therefore substantially enhance the detection of microorganisms, by adding potential for portability, cost affordability, and eventually automation. ${ }^{22}$ Optical waveguides and microfluidic channels have been embedded in polymer-based devices for fluorescence excitation of biological samples, such as fluorescently labeled phospholipids and stained cells. ${ }^{23}$ Another work reported a device based on a polydimethylsiloxane (PDMS) 2D optical lens integrated with microfluidic channels to improve the performance of fluorescence spectroscopy performed using optical fibers. ${ }^{24}$ Moreover, an optofluidic system based on a low-refractive index liquid with tunable biconcave PDMS lenses for focusing light has also been reported. ${ }^{25}$ Existing microfabrication techniques allow integration of optic and fluidic elements in lab-on-a-chip devices. ${ }^{26}$ Another work demonstrated a fluid-based microfabricated lens capable of integration into a multilens optical system for microfluidic flow cytometry. ${ }^{27}$ Another work presented microfluidic observation channel capable of performing flow cytometry measurements using solid-state lasers and PIN-based photodetectors used for fluorescent microsphere and nucleic acid labeled fungi. ${ }^{28}$ However, such approaches mainly employ expensive optics and fabrication methods, which may hinder the affordability one can aim at. In this work, in order to test the potential of empowering low-magnification systems for the analysis of microorganisms in a cost-effective fashion, we integrated $\mathrm{BaTiO}_{3}$ spheres in microstructured polymeric membranes toward the creation of microfluidic chips. With respect to previous works, our device constitutes a low-cost solution to potentially enhance the detection capability of low-end microscopy systems. The possibility of readily coupling polymeric membrane/dielectric sphere assembly (PMDSA) with an optical microscope makes the device possibly suitable for applications to on-site detection. Also, our method reduces manual work required for sample preparation steps by integrating the optical elements in a microfluidic tool, operated by programmable pumps. Moreover, embedding of dielectric spheres in the PDMS membrane is not limited by the design of the microchannels, which can be adapted according to the specific application.

\section{Methods}

\subsection{Analytical Modeling}

The procedure to calculate the optical path through the sphere has been described previously. ${ }^{3}$ Briefly, to quantitatively calculate the effect of the sphere on the light cone emerging from the sample, we used the property of cylindrical symmetry to reduce the calculation to a 1D problem and used linear wave propagation and Snell's law to compute the relation between the input and output angles relatively to the optical axis of the imaging system. By using the same notation as previously, ${ }^{3}$ the theoretical contrast gain was calculated as $\frac{1-\cos \left[\theta_{\mathrm{S}}(h)\right]}{1-\cos \left(\theta_{\mathrm{obj}}\right)}$, where $\theta_{\mathrm{obj}}$ is the maximum angle collected by the objective relatively to the optical axis, and $\theta_{\mathrm{S}}(h)$ is the maximum collected angle in presence of a 1-mm-diameter sphere at a distance $h$ from the sample along the optical axis.

\subsection{Chip Fabrication}

Microfluidic devices were prepared by soft lithography. Briefly, after surface activation (20 min in hexamethyldisilazane vapor) of 4-in. Si-wafers, we spin-coated $2 \mu \mathrm{m}$ of positive photoresist (AZ9260). We then exposed in hard-contact mode for $10 \mathrm{~s}\left(100 \mathrm{~mJ} / \mathrm{cm}^{2}\right)$ and developed the photoresist. After back-side rinsing, dry etching was achieved with the standard BOSCH process at $25^{\circ} \mathrm{C}$, with process time adjusted to obtain the desired height of the structure (etching rate 
$\approx 3.5 \mu \mathrm{m} / \mathrm{min}$ ). PDMS moulding mixture of 10:1 (prepolymer:curing agent) was mixed manually and degassed for $1 \mathrm{~h}$. The mixture obtained was then poured onto a Si-master or spincoated on a $\mathrm{Si}$ master depending on the desired structure. $\mathrm{BaTiO}_{3}$ spheres (Cospheric) were manually positioned on the PDMS film with tweezers. Curing was achieved by keeping at $100^{\circ} \mathrm{C}$ for $1 \mathrm{~h}$. The solid PDMS obtained was then peeled off and bonded to a PDMS block by standard plasma activation $(0.2 \mathrm{mbar} \mathrm{O} 2,3.0 \mathrm{~mA}, 0.5 \mathrm{kV})$ for $60 \mathrm{~s}$. Inlets and outlets were then manually drilled with a puncher, and finally the chip was bonded to a glass slide with the same plasma activation procedure.

\subsection{Image Acquisition and Processing}

The samples imaged consisted of Escherichia coli specimens genetically modified to express red-fluorescent protein (DsRed). Chip and bacteria were imaged with an Axio Imager M2 upright microscope, equipped with a SOLA light engine (SOLA SE 5-LCR-VB, Lumencor). A filter cube (Filter Set $43 \mathrm{HE}$, Zeiss) was placed in the optical path for fluorescence imaging. We used an air objective $10 \times / 0.25 \mathrm{NA}$ (Zeiss). Images were recorded with a sCMOS camera (pco.edge 4.2, Visitron Systems). Images were recorded in $2 \times 2$ binning mode, with variable exposure depending on the condition of the specific experiment. In order to automate the acquisition, the microfluidic chip was fixed on a motorized $x y z$-stage (Visitron) of an upright microscope and connected to a neMESYS syringe pump (Cetoni $\mathrm{GmbH}$ ). For the time series of fluorescence images recorded with flowing bacteria, the following processing was performed: the pixel-by-pixel median of the series was subtracted to each image to obtain background-free images; a Gaussian blur with $\sigma_{\mathrm{G}}=2$ was applied to each image (this step was performed only for images recorded in the presence of the sphere, where the $\approx 5.5 \times$ magnification increases the variability between adjacent pixels, which may bias the detection of a single bacterium by splitting it into several smaller particles); the standard deviation of the pixels of the entire series was calculated and a threshold was set to 10 times this value; each image was thresholded and the area and integrated intensity of each particle detected were calculated. For the time series of fluorescence images recorded with trapped bacteria, all images of the same sequence were aligned by a custom developed script based on template matching, ${ }^{29}$ in order to remove artifacts due to eventual movement along $x-y$ plane. Subsequently, two different regions of interest of the same area were drawn to define the immobilized bacterium and an adjacent region without for background evaluation. Finally, average grayscale values were measured in all the slices of the time series for both bacterium and background.

\subsection{Data Analysis}

Only the particles with an area higher than 20 pixels were considered, to prevent a potential bias from small particle artifacts. The values of the integrated intensity in the presence of the sphere were divided by $2 \pi \sigma_{\mathrm{G}}$ to correct for the Gaussian blur ${ }^{30}$ introduced upstream to ease the identification of the bacteria. All the values were then normalized to the highest one to ease comparison for different conditions. To compute the experimental CBR gain, we divided the values corresponding to the same exposure.

\section{Results}

\subsection{Embedding Highly Refractive Spheres in Microstructured Polymeric Membranes for Microfluidic Chips}

\subsubsection{Model}

Based on our previous resolution analysis on dielectric spheres immersed in homogenous media, ${ }^{3}$ we deduced the optimal configuration for a highly refractive sphere (barium titanate, refractive index $n_{S}=1.95$ ) embedded in a PDMS membrane (PDMS, refractive index $n_{\text {PDMS }}=$ 1.43) [Fig. 1(a)]: it consists of a sphere only partially embedded in PDMS, where the index 

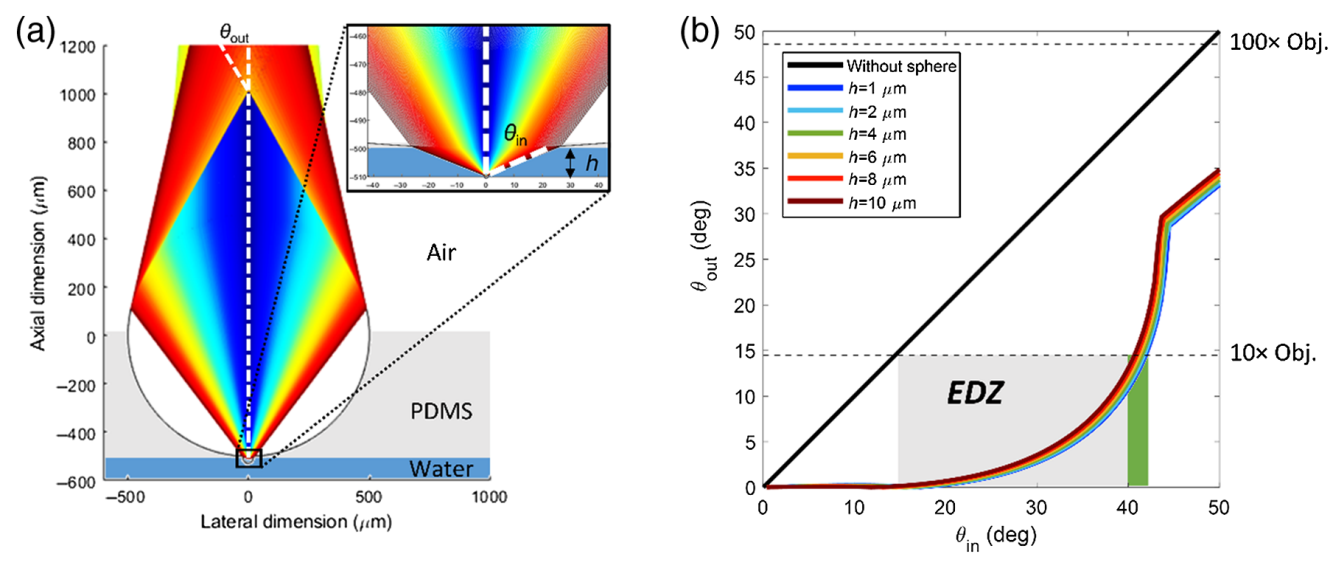

Fig. 1 Working principle of the PMDSA. (a) Rays emerge from a point source placed in a microfluidic channel and propagate upward through a $1-\mathrm{mm}$ barium titanate sphere partially embedded in PDMS up to the microscope objective (not shown). The sphere-sample distance is marked with $h ; \theta_{\text {in }}$ indicates the angle of a ray entering the sphere, whereas $\theta_{\text {out }}$ indicates the angle of the corresponding outcoming ray. The color gradient indicates different propagating rays. (b) Relation between $\theta_{\text {in }}$ and $\theta_{\text {out }}$ for the model shown in (a). The dashed lines mark the acceptance angle for a low-magnification (10x) and a high-magnification (100x) objective. The gray-shaded area indicates the EDZ, where the dielectric sphere mediates the collection of light coming from higher angles $\theta_{\text {in }}$ compared to the objective alone. The green-shaded area indicates the maximum angles that are collected by the resulting optical system, which corresponds to an effective objective with $\mathrm{NA} \approx 0.66$.

mismatch between the sphere material, the polymer, and the surrounding media increases the overall collection angle of the optical system $\left[\theta_{\text {out }}\right.$ in Fig. 1(b)]. This effect can be used to generate resolution and contrast gains for low-magnification objectives: in Fig. 1(b), we show the presence of an enhanced-detection zone (EDZ) for a $10 \times / 0.25 \mathrm{NA}$ objective, which approaches the collection angle of a $100 \times / 0.75 \mathrm{NA}$ objective with the same immersion medium (air).

\subsubsection{Fabrication of the PMDSA}

To simplify the fabrication of PMDSAs, we developed a procedure (Fig. 2) to create microstructured PDMS membranes with both customizable designs and embedded spheres to increase resolution and sensitivity. Initially, we patterned a 5-in. chromium-and-glass mask following the microchannels design. To fabricate the Si mold used for casting the PDMS sphere-embedded membrane, conventional photolithography was used to pattern a 1.5- $\mu \mathrm{m}$-thick layer of AZ9260 positive photoresist on a 4-in. 550- $\mu \mathrm{m}$-thick Si wafer. Subsequently, we dry-etched $6 \mu \mathrm{m}$ of the $\mathrm{Si}$ substrate with a $\mathrm{BOSCH}$ process and stripped the photoresist protective layer. We performed a silane treatment of the Si surface in order to prevent sticking of PDMS on the substrate during membrane peeling. To finally form the PMDSA, a 10:1 mix of PDMS prepolymer and curing agent was spin-coated on top of a microstructured $\mathrm{Si}$ wafer, to form a PDMS layer of $\approx 300 \mu \mathrm{m}$ thickness; then the spheres were manually positioned at the desired locations, and finally the PDMS was cured and peeled-off the Si mold. Pillars and hydrodynamic traps, located underneath the dielectric sphere, are spread over the microfluidic channels in dense arrays, so that positioning of the sphere does not require particular accuracy skills and can be easily performed manually by visual inspection with a stereomicroscope. The full chip was assembled by bonding the PMDSA to a thick and flat PDMS block or to a glass slide. Embedding of the $\mathrm{BaTiO}_{3}$ spheres directly in the patterned PDMS membrane highly improves the precision of the location of the spheres relative to the microfluidic structures compared to performing bonding between a patterned PDMS block and a membrane with embedded dielectric spheres: in the latter case, alignment is very sensitive because it must be done quickly after plasma activation of the PDMS surfaces and the bonding is irreversible; in the former procedure, spheres can be positioned very precisely before the curing step, which allows more time and reversible positioning. 


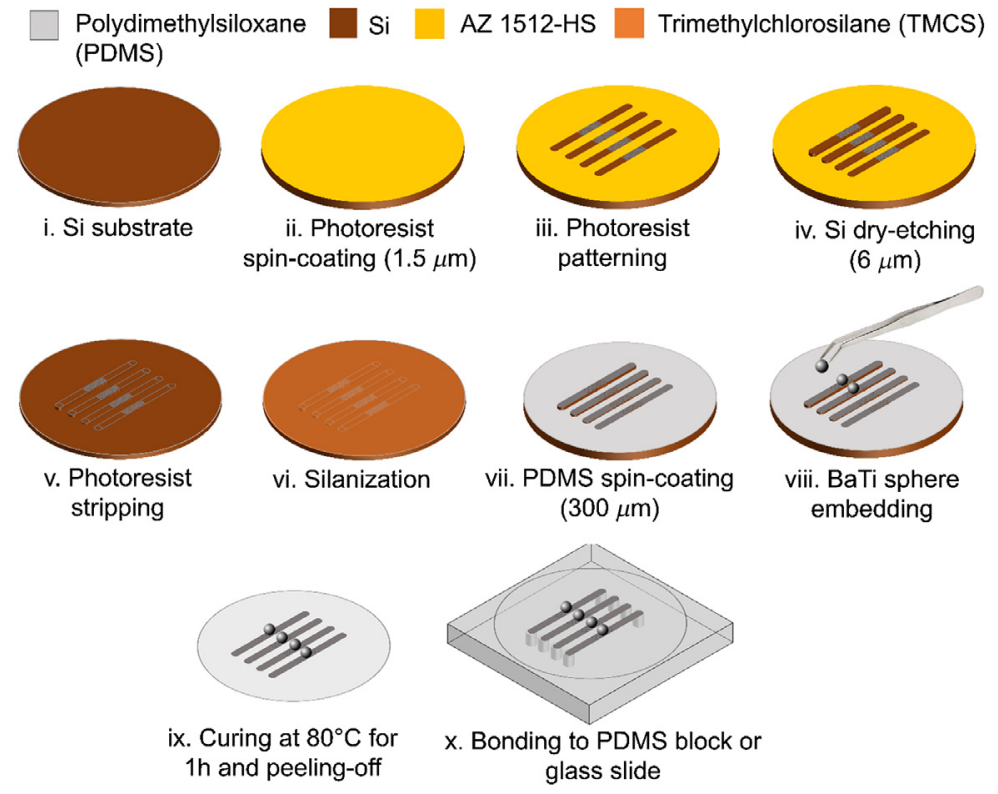

Fig. 2 Fabrication of the PMDSA. Schematic of the fabrication procedure of the PDMS membrane with partially embedded $\mathrm{BaTiO}_{3}$ dielectric sphere. (i)-(v) Channels and trapping structures are patterned on a Si wafer by photolithography and dry-etching processing. (vi) Silanization of the Si mold is performed to prevent PDMS from sticking to the wafer. (vii)-(ix) PDMS prepolymer is spin-coated and $\mathrm{BaTiO}_{3}$ dielectric spheres are manually placed at positions corresponding to the microfluidic structures. (ix), (x) The whole wafer is put at $80^{\circ} \mathrm{C}$ for PDMS curing, then the membrane with integrated spheres is peeled-off and bonded to a PDMS block or to a glass slide.

\subsubsection{Microfluidic chip design}

We fabricated a PMDSA with a design to analyze bacteria ( 1 to $2 \mu \mathrm{m}$ size) in aqueous media [Fig. 3(a)]. The device consisted of a 1-mm-large, 6- $\mu \mathrm{m}$-high channel with equally spaced cylindrical pillars [Fig. 3(b)]. The large and narrow channel keeps the hydraulic resistance low while keeping the bacteria close to the imaging region of the sphere. The pillars, $100 \mu \mathrm{m}$ in diameter, prevent the microchannel ceiling from collapsing [Fig. 3(c-i)]. The central region of the channel includes a large array of trapping structures intended for bacteria immobilization and long-term imaging [Fig. 3(c-ii)]. Trapping units have a sieve-like design and present a tapered opening of $1.2 \mu \mathrm{m}$ narrowing to $0.8 \mu \mathrm{m}$, for tight trapping of $E$. coli specimens. This type of confinement method allows to immobilize a small number of bacteria (down to a single bacterium) in each trapping unit: once a trap is filled, the flow passing through the sieve is stopped, preventing additional bacteria from being trapped. ${ }^{21}$ The packed array of traps built in the microchannel has also the functions to maximize the number of detected bacteria in the field of view of the dielectric sphere and to provide structural stability to the chip.

\subsection{Optical Performance of the Assembly}

\subsubsection{Contrast enhancement of a low-magnification system}

Since such chips can be used in a portable fashion and have the potential to be combined with simple optical detection systems, they could be used to detect pathogens in water basins in remote sites. To test the feasibility of this application, after an initial cleaning of the device by flowing a $70 \%$ ethanol solution, we injected red-fluorescent bacteria $(\approx 2000$ bacteria/ $\mu \mathrm{L})$ in the chip and recorded image sequences using a low-magnification objective $(10 \times / 0.25 \mathrm{NA})$. The detailed fluidic protocol utilized for this application is reported in Figs. 3(d-i). To assess the potential for fast image recording, we performed the analysis for short exposure (1 to $20 \mathrm{~ms}$ ). Moreover, since we do not require high resolution for such imaging, we used $2 \times 2$ binning to further decrease the acquisition time. After subtraction of the pixel-by-pixel median of the time 
(a)

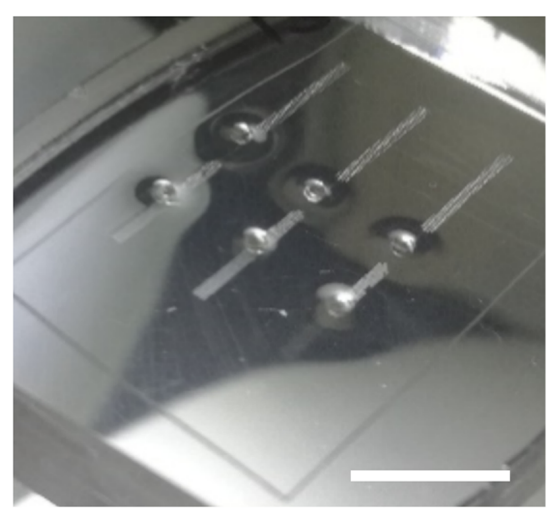

(c)

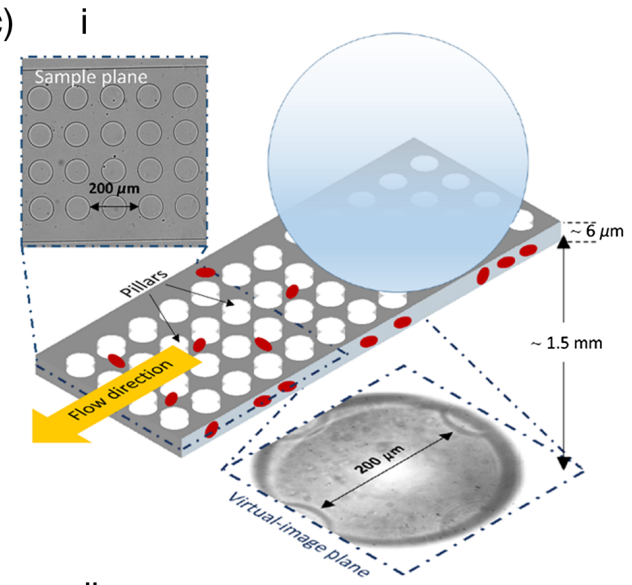

ii

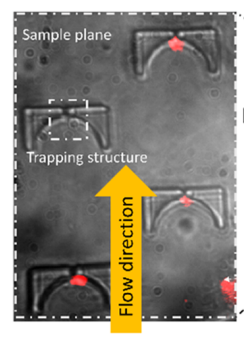

(b) $\mathrm{i}$
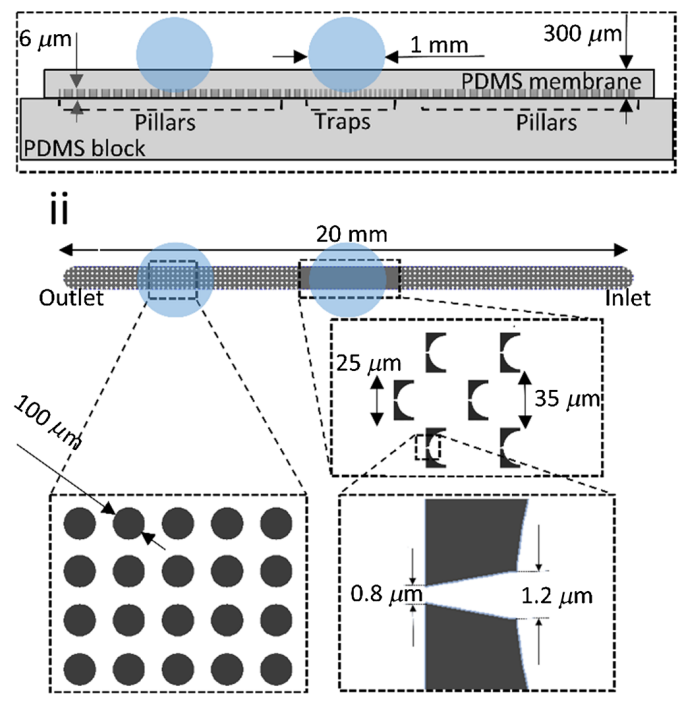

(d)
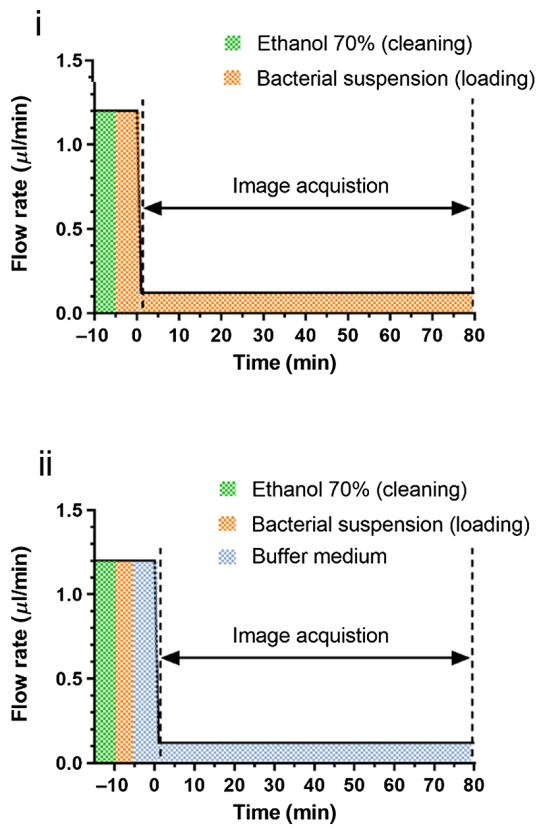

Fig. 3 Structure and operation of the microfluidic chip. (a) Picture of the microfluidic device bonded to a thick PDMS block. Scale bar: $1 \mathrm{~cm}$. (b) Vertical section (i) and top view (ii) schematics of the microfluidic chip with partially embedded $\mathrm{BaTiO}_{3}$ spheres. (c) Schematics of microstructured PDMS membranes with partially embedded spheres, bonded to a thick PDMS block to create a channel for imaging of bacteria in a flow (i) or in hydrodynamic traps (ii). In order to sustain the membrane and avoid collapsing of the top to the bottom of the channel, PDMS pillars are embedded in the microchannel. Zooms of the functional units of the chip with bright-field clichés are indicated by dash-and-dot outlines. Red fluorescent signal from trapped bacteria is overlapped to the bright-field cliché of the traps. (d) Microfluidic protocols used during imaging of fluorescent bacteria in a flow (i) and in traps (ii).

series to each frame, we obtained background-free images [Fig. 4(a)]. The PMDSA introduces a $\approx 5.5 \times$ magnification but also some aberrations which distort the expected oval shape of bacteria. By color-coding the different time frames, one can obtain the trajectories of the bacteria in the channel [Fig. 4(a-ii)]. The contrast-to-background ratio (CBR) was quantified as the ratio of the average signal over the average of the background, and finally normalized to its maximum experimental realization. In Fig. 5(a), we report the normalized CBR measured for several exposures. As expected, for short exposure time, it increases with the exposure for both the conditions 
(a)
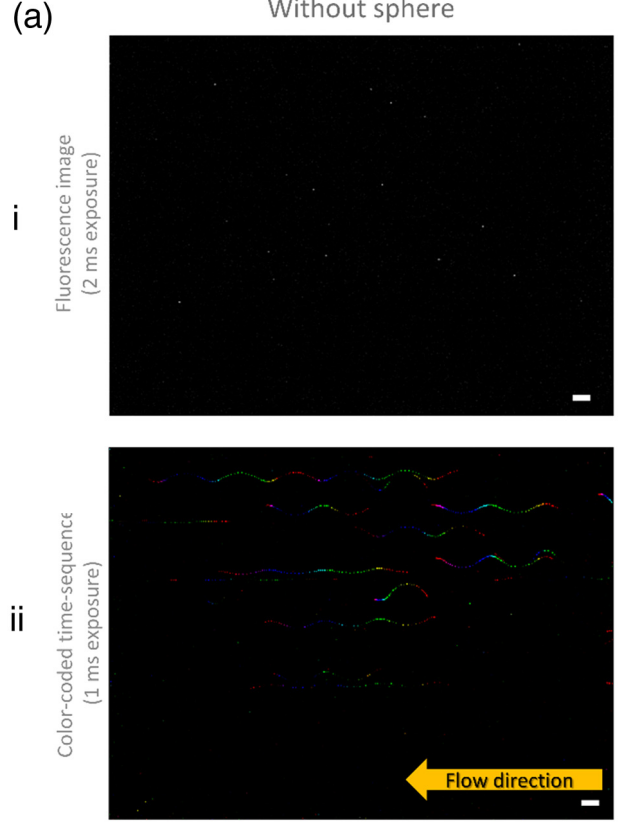

(b) $\mathrm{i}$

$0 \mathrm{~min}$

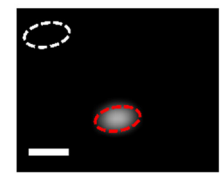

$40 \mathrm{~min}$

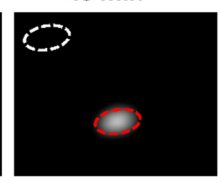

$80 \mathrm{~min}$

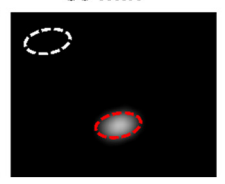

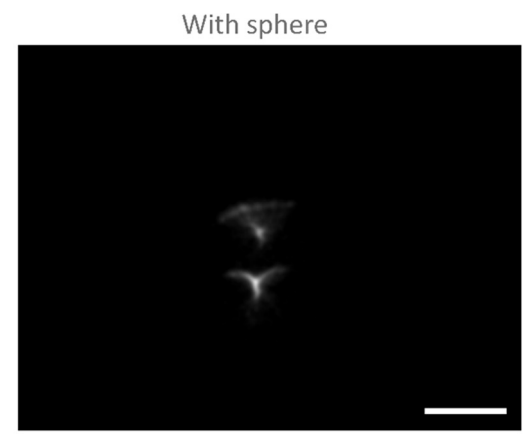
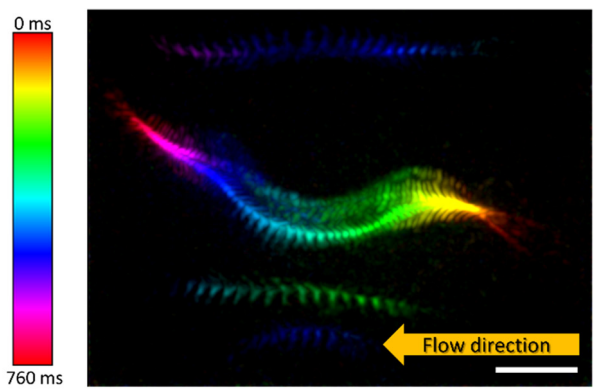

ii
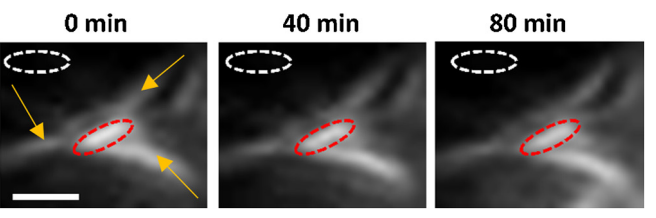

Fig. 4 Imaging of living fluorescent bacteria. (a) Fluorescence images (i) and color-coded time sequences (ii) of a flowing suspension of fluorescent bacteria imaged with a low-magnification objective $(10 \times / 0.25 \mathrm{NA})$ in either absence or presence of the dielectric sphere. Scale bars: $10 \mu \mathrm{m}$. (b) Time-lapse fluorescence of a single trapped bacterium imaged with a high-magnification objective $(100 \times / 0.75 \mathrm{NA})$ (i) and with a low-magnification objective $(10 \times / 0.25 \mathrm{NA})$ in the presence of the membrane with partially embedded $\mathrm{BaTiO}_{3}$ sphere (ii). Scale bars: $2 \mu \mathrm{m}$. Red outline: region containing the least-aberrated fluorescent signal of the bacterium. White outline: region containing the reference background. Arrows indicate aberrations introduced by the partially embedded spheres.

[Fig. 5(a), dark gray area]. Additional data obtained for higher exposure (made possible with immobilized bacteria as discussed in the next section) show the plateauing effect for which even a one-order-of-magnitude increase in the exposure does not result in a significant increase of the CBR [Fig. 5(a) light gray area]. The CBR gain for the low-magnification objective in the presence of the PMDSA was then calculated and compared to the theoretical gain predicted by the model [Fig. 5(b)].

\subsubsection{Comparison with a high-magnification system}

In order to assess the performances of the PMDSA coupled to a low-magnification objective $(10 \times / 0.25 \mathrm{NA})$ compared to a high-magnification system $(100 \times / 0.75 \mathrm{NA})$, we trapped fluorescent bacteria in a microfluidic channel and imaged them with both systems. For this purpose, we utilized an array of hydrodynamic traps designed to confine the bacterial cells during the image acquisition [Fig. 3(c)]. The trapping section is positioned at the centre of each microchannel underneath the dielectric sphere. The fluidic protocol used for the experiment is shown in detail in Fig. 3(d-ii). After a cleaning step, a suspension of fluorescent bacteria was injected into the channels. Afterward, buffer medium was injected to only retain the stably trapped bacteria. Finally, we set a constant injection flow rate at $0.12 \mu \mathrm{L} / \mathrm{min}$ in order to avoid the escape of confined bacteria from immobilization sites during time-lapse imaging, performed over $80 \mathrm{~min}$ 
(a)

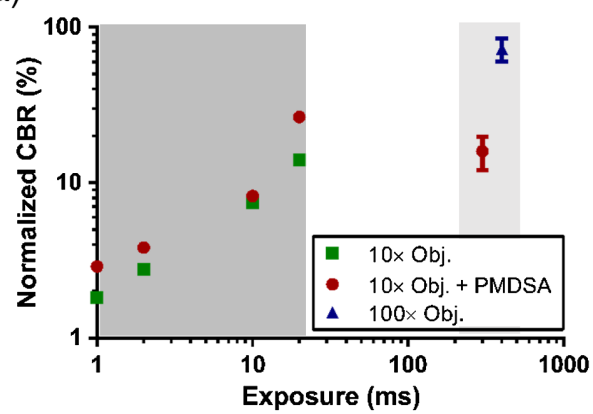

(b)

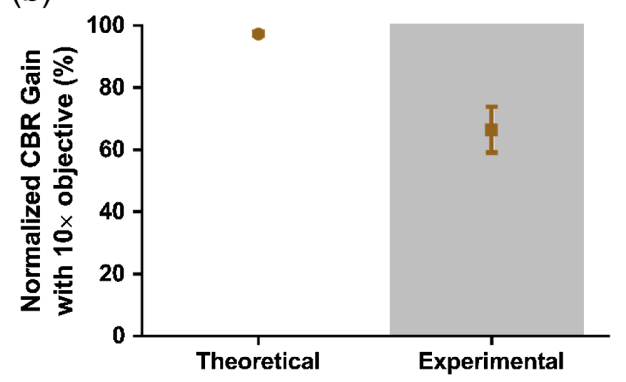

(c)

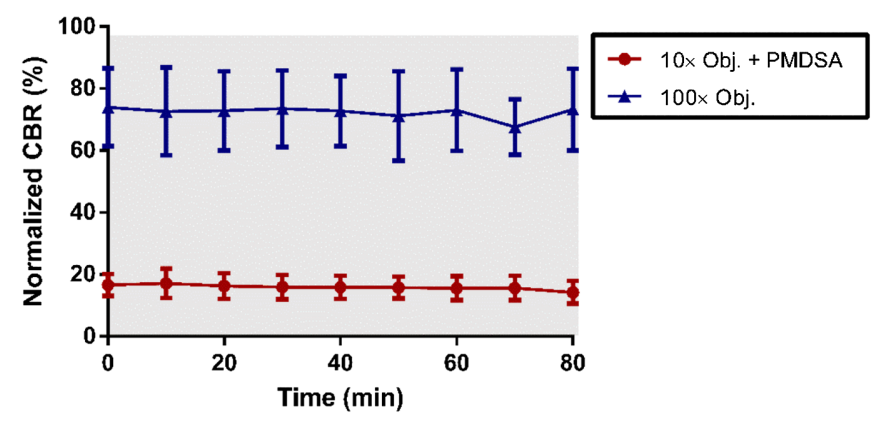

(d)

\begin{tabular}{|c|c|c|c|c|c|c|}
\hline & Magnification & NA & $\begin{array}{l}\text { Free working } \\
\text { distance }(\mathrm{mm})\end{array}$ & Immersion & $\begin{array}{l}\text { Field of view } \\
(\mathrm{mm})\end{array}$ & Price [\$\$] \\
\hline $\begin{array}{c}\text { Objective LD EC } \\
\text { epiplan-neofluar } \\
100 x / 0.75 \text { DIC M27 }\end{array}$ & $100 x$ & 0.75 & 4 & $\begin{array}{c}\text { Without } \\
\text { immersion }\end{array}$ & 25 & $\approx 6300$ \\
\hline $\begin{array}{l}\text { Objective EC epiplan- } \\
\text { neofluar } 10 x / 0.25 \text { DIC } \\
\text { M27 }\end{array}$ & $10 x$ & 0.25 & 9.3 & $\begin{array}{l}\text { Without } \\
\text { immersion }\end{array}$ & 25 & $\approx 2300$ \\
\hline
\end{tabular}

Fig. 5 Quantitative assessment of the optical enhancement. (a) Normalized CBR as a function of exposure time for several optical configurations. Data are plotted as mean $\pm \operatorname{SEM}(n \geq 3)$. The gray-shaded areas indicate the corresponding data plotted in (b) and (c). (b) Experimental and theoretical CBR gain. (c) Normalized CBR of trapped bacteria over time. Data are plotted as mean \pm SEM $(n=3)$. (d) Summary of the main features of the low- and high-magnification objectives used. Shaded areas indicate the correspondence between the values in the different graphs.

[Fig. 4(b)]. The fluorescence signal was quantified by measuring the average intensity value inside the region of interest delimited by the elliptic shape of the bacterium. The background value was quantified as the average intensity value inside a region of interest with the same area as the one used for the bacterium but positioned away from the trap. Comparison between normalized CBR measured for the PMDSA coupled with a low-magnification objective $(10 \times / 0.25 \mathrm{NA})$ with respect a high-magnification system $(100 \times / 0.75 \mathrm{NA})$ is illustrated in Fig. 5(c). The CBR resulted to be stable over time for both the systems, which show that the structure composed by the thin membrane with embedded sphere does not introduce mechanical instabilities which could result in drift or oscillations of the focal plane. Conversely, the CBR of the high-magnification objective remains 3.5 larger than the one obtained with the polymeric membrane with embedded $\mathrm{BaTiO}_{3}$ sphere, which is not surprising when considering the huge difference in terms of optimization and corresponding cost [Fig. 5(d)].

\section{Conclusion}

We have described a method to enhance the contrast in low-magnification optical detection based on highly refractive $\mathrm{BaTiO}_{3}$ spheres partially embedded in polymeric membranes, which 
we integrated on microfluidic chips for the imaging of living fluorescent bacteria. The present simple fabrication procedure enables to create custom designs for the chips and, at the same time, to precisely locate the spheres relative to the microfluidic structures.

The ability of the PMDSA to enhance the detection of bacteria in a continuous flow already for short exposure [Fig. 5(a), dark gray area] paves the way for applications where flow cytometry needs to be performed in remote sites, where bulky equipment should be suitably replaced by portable optical and microfluidic systems. Moreover, we demonstrated the opportunity to integrate specific functional microfluidic elements (e.g., trapping structures). Such customizations can guarantee reliable long-term imaging capabilities [Fig. 5(c)] and thus potentially enable applications such as antibiotic testing directly on site.

By comparing the experimental results with the theoretical calculation for the PMDSA [Fig. 5(b)], we observe that the system is suboptimal $(\approx 30 \%$ lower than the expected theoretical gain). This loss may be due to several factors that can cause a disruption in the light path from the sample to the objective:

- the inhomogeneities in the sphere size and material;

- the aberrations introduced by the sphere [Fig. 4(b-ii), arrows];

- the reduced depth-of-field in presence of the sphere $\frac{\lambda_{\text {em }} n}{\mathrm{NA}^{2}} \approx \frac{584 \mathrm{~nm} \cdot 1}{0.66^{2}}=1.3 \mu \mathrm{m}$ (which is comparable to the size of a bacterium and thus can exclude some light coming from it from being collected by the objective) compared to the conventional condition $\frac{\lambda_{\mathrm{em}} n}{\mathrm{NA}^{2}} \approx$ $\frac{584 \mathrm{~nm} \cdot 1}{0.25^{2}}=9.3 \mu \mathrm{m}$.

Moreover, despite the focusing effect from the sphere (which increases the contrast gain), the excitation through the sphere may also be suboptimal because of:

- the inhomogeneities in the sphere size and material;

- internal reflexion at its bottom side for a part of the excitation light.

Given the nature of the PMDSA proposed here, we expect spherical aberration to be present because of the presence of the $\mathrm{BaTiO}_{3}$ sphere, and we cannot exclude the presence of other aberrations. Astigmatism or coma for instance can be introduced by imperfections in the shape of the dielectric sphere. All the aberrations introduced by the addition of the PMDSA to the optical system degrade the image of the object, which can be certainly detected with higher confidence, but at the price of lowering resolution on its details. This system is therefore more adapted for optical signal detection (because of increased contrast) rather than for complete imaging (which usually requires higher resolution). To obtain quantitative information about the aberrations introduced by the PMDSA, one may study the aberrated point-spread function in terms of its decomposition with the Zernike polynomials, which analytically describe the different types of aberrations. ${ }^{31}$ This may be accomplished by imaging fluorescent nanoparticles, which can be safely approximated as point sources, which cannot be done for the bacteria in our study. Models are also used to analyze and correct distortion, ${ }^{32,33}$ which can be needed especially when parts of the object are imaged far from the center of the sphere, which has a total field of view of about $0.03 \mathrm{~mm}^{2}$. Once the aberrations are more quantitatively described, one may use hardware and software solutions to obtain higher-resolution images. From a hardware point of view, one can design the shape of the polymeric membrane (which is flat in the imaging zone for simplicity of fabrication in our study) in order to compensate for some aberrations. This method is often used in commercial objectives, where several lenses altogether provide much less aberrations than the individual ones. From a software point of view, one can apply deconvolution algorithms to the aberrated images. Such methods can highly increase the net resolution of the PMDSA system.

The problem of fabrication costs is well known in microfluidics, a domain which still enormously relies on clean-room microfabrication techniques, which are highly expensive. In fact, everything intrinsically depends on the type of structure one wants to create in the chip. Assuming that conventional photolithography techniques are used for Si wafer processing, the cost of fabrication of a mold is of the order of $100 \$$ (material + expenses on clean-room equipment only). A Si master can be used multiple times for PMDSAs casting, such method of production allowing to reduce the cost of a single PMDSA unit. Fabrication expenses are mainly 
related to material costs of PDMS together with dielectric spheres (0.5 \$/PMDSA) and of utilization of PDMS processing equipment $(30 \$ / \mathrm{h})$. However, other methods besides clean-room microfabrication are applicable to obtain reliable chips with lower costs in terms of infrastructure and material. Injection-molding, for instance, would be ideal for large scale production of chips with the same design. Given the low cost of such dielectric spheres (0.04 \$/sphere) and the current continuous decrease of the fabrication methods for microfluidic chips (such as hotembossing and injection-moulding ${ }^{34}$ ), we believe that the proposed fabrication protocol is readily adaptable to a wide variety of microfluidic chips with integrated optical elements. After initial investment in the creation of the mold (which may rise up to tens of $\mathrm{k} \$$ ), the cost of a single chip may be lowered to $1 \$$ or less, depending on the fabrication material used. Moreover, with a suitable mold, one may eventually improve the positioning of the dielectric spheres on the chip and decrease the time spent for this operation as well. When considered together with the potential cost reduction associated to low-end imaging systems, the present approach has the potential to highly increase the usability in low-resource settings for the often required on site analyses.

\section{Acknowledgments}

This work was supported by the Swiss National Science Foundation (No. 205321-179021). We thank the staff of the Center of Micro- and Nanotechnology of EPFL for assistance in the microfabrication processes. The authors declare no conflicts of interests.

\section{References}

1. A. Darafsheh et al., "Optical super-resolution by high-index liquid-immersed microspheres," Appl. Phys. Lett. 101, 141128 (2012).

2. S. Lee et al., "Immersed transparent microsphere magnifying sub-diffraction-limited objects," Appl. Opt. 52, 7265-7270 (2013).

3. D. Migliozzi, M. A. M. Gijs, and G. Huszka, "Microsphere-mediated optical contrast tuning for designing imaging systems with adjustable resolution gain," Sci. Rep. 8, 15211 (2018).

4. A. Darafsheh et al., "Optical super-resolution imaging by high-index microspheres embedded in elastomers," Opt. Lett. 40, 5-8 (2015).

5. B. Yan et al., "Superlensing microscope objective lens," Appl. Opt. 56, 3142-3147 (2017).

6. M. Duocastella et al., "Combination of scanning probe technology with photonic nanojets," Sci. Rep. 7, 3474 (2017).

7. H. Yang et al., "Super-resolution biological microscopy using virtual imaging by a microsphere nanoscope," Small 10, 1712-1718 (2014).

8. F. Wang et al., "Scanning superlens microscopy for non-invasive large field-of-view visible light nanoscale imaging," Nat. Commun. 7, 13748 (2016).

9. J. Li et al., "Swimming microrobot optical nanoscopy," Nano Lett. 16, 6604-6609 (2016).

10. L. Li et al., "Label-free super-resolution imaging of adenoviruses by submerged microsphere optical nanoscopy," Light Sci. Appl. 2, e104 (2013).

11. J. J. Schwartz, S. Stavrakis, and S. R. Quake, "Colloidal lenses allow high-temperature single-molecule imaging and improve fluorophore photostability," Nat. Nanotechnol. 5, 127-132 (2010).

12. H. Yang, M. Cornaglia, and M. A. M. Gijs, "Photonic nanojet array for fast detection of single nanoparticles in a flow," Nano Lett. 15, 1730-1735 (2015).

13. P. Ghenuche et al., "Multi-focus parallel detection of fluorescent molecules at picomolar concentration with photonic nanojets arrays," Appl. Phys. Lett. 105, 131102 (2014).

14. H. Aouani et al., "High-efficiency single molecule fluorescence detection and correlation spectroscopy with dielectric microspheres," Proc. SPIE 7571, 75710A (2010).

15. G. Huszka, H. Yang, and M. A. M. Gijs, "Microsphere-based super-resolution scanning optical microscope," Opt. Express 25, 15079-15092 (2017).

16. G. Huszka and M. A. M. Gijs, "Turning a normal microscope into a super-resolution instrument using a scanning microlens array," Sci. Rep. 8, 601 (2018). 
17. K. W. Allen et al., "Super-resolution microscopy by movable thin-films with embedded microspheres: resolution analysis," Ann. Phys. 527, 513-522 (2015).

18. Y. Hennequin et al., "Optical detection and sizing of single nano-particles using continuous wetting films," ACS Nano 7, 7601-7609 (2013).

19. G. Huang et al., "Improving axial resolution for holographic tracking of colloids and bacteria over a wide depth of field by optimizing different factors," Opt. Express 26, 9920-9930 (2018).

20. D. D. Carlo, L. Y. Wu, and L. P. Lee, "Dynamic single cell culture array," Lab Chip 6(11), 1445 (2006).

21. M.-C. Kim et al., "Programmed trapping of individual bacteria using micrometre-size sieves," Lab Chip 11, 1089-1095 (2011).

22. H. Yang and M. A. M. Gijs, "Micro-optics for microfluidic analytical applications," Chem. Soc. Rev. 47, 1391-1458 (2018).

23. M. Schelb et al., "Fluorescence excitation on monolithically integrated all-polymer chips," J. Biomed. Opt. 15, 041517 (2010).

24. S. Camou, H. Fujita, and T. Fujii, "PDMS 2D optical lens integrated with microfluidic channels: principle and characterization," Lab Chip 3, 40-45 (2003).

25. C. Song et al., "Biconcave micro-optofluidic lens with low-refractive-index liquids," Opt. Lett. 34, 3622-3624 (2009).

26. S. M. Azmayesh-Fard, E. Flaim, and J. N. McMullin, "PDMS biochips with integrated waveguides," J. Micromech. Microeng. 20, 087002 (2010).

27. J. Godin, V. Lien, and Y.-H. Lo, "Demonstration of two-dimensional fluidic lens for integration into microfluidic flow cytometers," Appl. Phys. Lett. 89, 061106 (2006).

28. Y.-C. Tung et al., "PDMS-based opto-fluidic micro flow cytometer with two-color, multi-angle fluorescence detection capability using PIN photodiodes," Sens. Actuators B 98, 356-367 (2004).

29. Q. Tseng, "Template matching and slice alignment," ImageJ Plugins, sites.google.com/site/ qingzongtseng/template-matching-ij-plugin (2011).

30. E. S. Gedraite and M. Hadad, "Investigation on the effect of a Gaussian blur in image filtering and segmentation," in Proc. ELMAR (2011).

31. V. N. Mahajan and J. A. Diaz, "Imaging characteristics of Zernike and annular polynomial aberrations," Appl. Opt. 52, 2062-2074 (2013).

32. D. C. Brown, "Decentering distortion of lenses," Photogramm. Eng. 32, 444-462 (1966).

33. J. E. Greivenkamp, Field Guide to Geometrical Optics, SPIE Press, Bellingham, Washington (2004).

34. V. Faustino et al., "Biomedical microfluidic devices by using low-cost fabrication techniques: a review," J. Biomech. 49, 2280-2292 (2016).

Vittorio Viri received his BSc degree in clinical engineering in 2013 and his MSc degree in biomedical engineering in 2016 from the University of Rome "La Sapienza," Italy. He is currently a doctoral assistant at EPFL, Switzerland. His research interests are in the development of microfluidic tools for studies on C. elegans nematodes and biological applications.

Daniel Migliozzi received his BSc degree in engineering physics from the Politecnico di Milano, Italy, in 2011, his double MSc degrees in biophysics and bioengineering from EPFL, Switzerland and Ecole Polytechnique, France, in 2014, and his PhD in microsystems from EPFL in 2019. He worked on research projects in collaboration with corporations such as CEA-LETI, France, and Nestlé, Switzerland, and with start-ups such as Lunaphore Technologies and Nagi Bioscience, Switzerland. He holds a double position as a scientist at EPFL and as an R\&D engineer at the Swiss Centre of Electronics and Microtechnology candidate at EPFL. His technical interests include optical and microfluidic technologies for applications in life sciences.

Martin A. M. Gijs received his degree in physics from Katholieke Universiteit Leuven, Belgium, in 1981, and his PhD in physics from the same university in 1986. He joined the Philips Research Laboratories at Eindhoven, Netherlands, in 1987. He joined EPFL in 1997. His present interests are in developing technologies for innovative magnetic devices, microfabrication technologies for microsystems fabrication in general, and the development and use of microsystems technologies for microfluidic and biomedical applications in particular. 